BMJ Open Sport \& Exercise Medicine

\title{
The effect of 1 week of repeated ischaemic leg preconditioning on simulated Keirin cycling performance: a randomised trial
}

\author{
Angus Lindsay, ${ }^{1,2}$ Carl Petersen, ${ }^{1}$ Gavin Blackwell, ${ }^{1}$ Hamish Ferguson, ${ }^{3}$ \\ Greg Parker, ${ }^{4}$ Nina Steyn, ${ }^{4}$ Steven P Gieseg ${ }^{4,5}$
}

To cite: Lindsay A, Petersen C, Blackwell G, et al. The effect of 1 week of repeated ischaemic leg preconditioning on simulated Keirin cycling performance: a randomised trial. BMJ Open Sport Exerc Med 2017;3: e000229. doi:10.1136/ bmjsem-2017-000229

- Additional material is published online only. To view please visit the journal online (http://dx.doi.org/10. 1136/bmjsem-2017-000229)

Accepted 26 March 2017

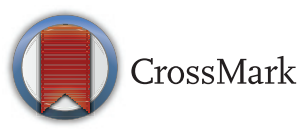

${ }^{1}$ School of Health Sciences, University of Canterbury, Christchurch, New Zealand ${ }^{2}$ Program in Physical Therapy and Rehabilitation Sciences, University of Minnesota, Minneapolis, USA ${ }^{3}$ Sport Performance Research Institute, Auckland University of Technology, Auckland, New Zealand ${ }^{4}$ School of Biological Sciences, University of Canterbury College of Science, Christchurch, New Zealand

${ }^{5}$ Department of Radiology, University of Otago Christchurch, Christchurch, New Zealand

Correspondence to Dr Carl Petersen; carl. petersen@canterbury.ac.nz

\section{ABSTRACT}

Background Coaches continually seek new ways of doing things and also refine existing techniques to improve sporting performance. Coaches are currently experimenting using ischaemic preconditioning (IPC) over consecutive days in the hope of improving competitive performances.

Aims First, to quantify the physiological impact of 1 week of IPC on simulated Keirin cycling performance. Second, to investigate if biochemical stress markers are affected over the treatment period.

Methods Using a randomised, sham-controlled design, 18 active adults undertook seven consecutive days of IPC treatment $(4 \times 5 \mathrm{~min}$ occlusion/reperfusion) applied to each leg at either $220 \mathrm{~mm} \mathrm{Hg}$ (treatment, $\mathrm{n}=9$ ) or $20 \mathrm{~mm} \mathrm{Hg}$ (sham, $\mathrm{n}=9$ ). Urinary measures of inflammation, oxidative stress and indirect nitric oxide synthesis were undertaken daily. A simulated Keirin cycling competition $(4 \times 30 \mathrm{~s}$ Wingate tests) was performed on day 10 , with baseline and postintervention cycling V02max (days 1, 11 and 18) and $30 \mathrm{~s}$ Wingate tests (day 2) undertaken for comparison.

Results The treatment group had enhanced mean cycling power (3.4\%), while neopterin and biopterin in conjunction with total neopterin were significantly lower $(p<0.05)$ and total biopterin significantly greater $(p<0.05)$ during the simulated Keirin. Aerobic fitness measures significantly improved from baseline to postintervention (VO2peak: 12.8\% $\uparrow$, maximal aerobic power: $18.5 \% \uparrow$ ).

Conclusions Seven consecutive days of IPC improved aerobic and anaerobic capacity measures, with modulations in oxidative stress, immune system activation and nitric oxide/catecholamine synthesis.

\section{BACKGROUND/AIM}

Coaches seek new ways to improve sporting performance, and considerable research has focused on pre-exercise interventions. ${ }^{1}{ }^{2}$ Repetition of using pressure to induce brief ischaemia (hypoxia) followed by reperfusion (reoxygenation) is known as ischaemic preconditioning (IPC). This technique can increase exercise capacity through alterations in oxygen delivery, blood flow and fatigue attenuation, ${ }^{3}$ and results in improved peak oxygen uptake ${ }^{4}$ and peak and mean power during the early stages of repeated sprint cycling. ${ }^{5}$ There is currently insufficient experimental proof to draw confident conclusions about the potential of IPC to acutely enhance sports performance, ${ }^{6}$ and this may be explained by respondents and non-respondents. ${ }^{6}$ If a single episode of IPC does enhance performance, would repeated exposure over several days induce an even greater effect? One recent study repeated IPC over nine consecutive days but failed to see any improvement in aerobic capacity. ${ }^{7}$ Repeated IPC has been investigated in clinical studies, with local and remote improvements in endothelial function, brachial artery flowmediated dilation and resting skin microcirculation being observed, ${ }^{8-10}$ although the physiological basis of this 'hyperconditioning' is still to be fully elucidated. ${ }^{11}$

Oxidative stress is an integral component of ischaemia/reperfusion, ${ }^{12}$ while vasodilation improved tissue oxygenation, ${ }^{13}{ }^{14}$ and inflammatory system modulation ${ }^{15}$ is regulated through single or repetitive ischaemic treatment. Neopterin, 7,8-dihydroneopterin, biopterin, 7,8-dihydrobiopterin and tetrahydrobiopterin are heterocyclic pterin compounds metabolised by various cell types whose detection and elevation represent perturbations in oxidative stress, immune system activation and nitric oxide/ catecholamine synthesis. ${ }^{16-21}$ Collectively, measurement of these biomarkers should provide some physiological insight into the stress response associated with repeated IPC.

Our research purpose is to assess induced physiological changes and simulated Keirin 
cycling performance following 7 days of IPC, and seek to determine if any aerobic capacity changes remain after an additional 7 days. We also aim to determine if urinary pterins are affected by the repeated ischaemiareperfusion tissue exposure during treatment as well as during exercise.

\section{METHODS}

\section{Participants}

Eighteen recreationally active (exercising $5.1 \pm 1.7$ hours/week) sport science students (13 men, 5 women; age: $23.2 \pm 7.1$ year; height: $177 \pm 11.4 \mathrm{~cm}$; weight: $79.2 \pm 12.7 \mathrm{~kg}$; lean body mass: $62.4 \pm 11.4 \mathrm{~kg}$; VO2max: $38.6 \pm 5.9 \mathrm{~mL} / \mathrm{min} / \mathrm{kg}$ ) volunteered for the study. All procedures performed were in accordance with the ethical standards of the University of Canterbury Human Ethics Committee. Informed consent was obtained from all individuals.

\section{Experimental design}

A randomised, sham-controlled, single-blinded (researchers only) design was used to assess the impact of seven consecutive days of IPC sessions on urinary markers of physiological stress and simulated competitive cycling performance measured through repeated anaerobic exercise performances. A power analysis for urinary pterins indicated that we needed nine or more subjects using a power of 80 and $\mathrm{p}$ value $<0.05$.

Baseline aerobic (VO2max) and anaerobic capacity (30 s Wingate) tests were performed 48 and 24 hours, respectively, prior to commencing the IPC protocol. Using relative VO2max, participants were pairmatched by the lead investigator, and using a coin toss were randomly allocated into treatment or 'sham' group. The participants were told that it was an IPC study using blood pressure cuffs; however, they were not told what pressure would be expected to be an effective treatment. Furthermore, the participants were unaware as to which treatment group they were in. The prescribed IPC sessions were performed once a day over the following 7 days. Participant characteristics are provided in table 1. Urine samples were collected (midstream) $5 \mathrm{~min}$ before each IPC session and stored on ice after collection and subsequently stored at $-80^{\circ} \mathrm{C}$ until analysis for markers of inflammation, oxidative stress and indirect nitric oxide/catecholamine synthesis. After completing all seven IPC treatments, the participants performed a simulated Keirin $(4 \times 30 \mathrm{~s}$ Wingate tests; 24 hours post) and a second and third VO2max test (48 hours, 7 days post).

\section{Methodology}

All participants visited the laboratory $\left(\sim 20^{\circ} \mathrm{C}\right.$; humidity $51 \%$ ) on 12 separate occasions. On the first day, using a Cyclus 2 ergometer (Leipzig, Germany) and Cortex Metalyzer 3B (Biophysik, Leipzig, Germany) metabolic cart, participants performed a progressive VO2max
Table 1 Subject characteristics

Treatment

Sham

$(n=9,6 M ; 3 W)$

(n=9, 7M;2W)

\begin{tabular}{lrr}
\hline Age (years) & $21.3 \pm 5.8$ & $26.1 \pm 8.8$ \\
\hline Height $(\mathrm{cm})$ & $177.3 \pm 4.4$ & $176.2 \pm 3.4$ \\
Weight $(\mathrm{kg})$ & $76.6 \pm 4.4$ & $81.7 \pm 4.1$ \\
VO2max $(\mathrm{mL} / \mathrm{min} / \mathrm{kg})$ & $38.2 \pm 1.9$ & $39.0 \pm 2.1$ \\
Pre-IPC peak power $(\mathrm{W})$ & $988 \pm 300$ & $1063 \pm 122$ \\
\hline
\end{tabular}

Data are presented as mean $\pm \mathrm{SD}$.

$\mathrm{IPC}$, ischaemic preconditioning; $\mathrm{M}$, men; $\mathrm{W}$, women; VO2max, relative VO2 max.

protocol starting at $100 \mathrm{~W}$ with $25 \mathrm{~W} / \mathrm{min}$ increments until exhaustion. A $30 \mathrm{~s}$ Wingate test (external drop weight equivalent to $10 \%$ body weight) performed on a Monark Ergometer (Ergomedic 894E, Vansbro, Sweden) was undertaken 24 hours later following a standardised 13 min warm-up. Participants received no verbal encouragement. Fingertip blood lactate capillary samples (LactatePro, ARKRAY, Kyoto, Japan) were collected pre, immediately and $5 \mathrm{~min}$ post Wingate test. Participants completed an adapted visual analogue scale (VAS) questionnaire $(0-10)$ of fatigue ${ }^{22}$ pre, post and $30 \mathrm{~min}$ post. A ' 0 ' represented 'no fatigue' while a ' 10 ' represented 'greatest fatigue possible'. While no familiarisation testing was performed before the baseline measurements, the participants were sport science students and have previously undertaken this type of testing. Nevertheless, the participants have had limited experienced with the particular testing, and we acknowledge that this is a limitation of the current study.

After baseline data collection, all participants underwent either 7 days of daily sham or IPC treatments. Wingate performance was retested 24 hours after the final IPC session during an anaerobic simulated cycling event (Keirin). The purpose of the simulated Keirin event was to understand the effect of repeated IPC on repetitive anaerobic exercise, which is common to many sports. The Keirin is a $2000 \mathrm{~m}$ motor-paced velodrome cycling event where competitors sprint the final $2 \frac{1}{2}$ laps $(625 \mathrm{~m})$ comprising $\sim 30 \mathrm{~s}$ of anaerobic effort. A typical Keirin competition involves two morning races an hour apart with two final races 6 and 7 hours later. Using four Wingate tests, we replicated this time scale and simulated a Keirin competition. Blood lactate was collected before, immediately post and $5 \mathrm{~min}$ post each Wingate, while urine was collected pre, immediately post and $30 \mathrm{~min}$ post each Wingate test, as well as 24 hours post. Peak power (W), average power (W) and fatigue index (\%) ([(peak power-finishing power)/peak power $\times 100$ ) were calculated for each Wingate while participants also completed the same VAS questionnaire pre, post and $30 \mathrm{~min}$ post. 
VO2max testing was conducted 48 hours and 7 days following the last IPC session. Each participant was asked to remain in a euhydrated state and adhere to their normal diet and activities throughout the 2 weeks of testing while also providing a training diary for the duration of the studies. Throughout the study, participants performed their normal training activities (exercising 5.1 \pm 1.7 hours/week). Participants were instructed to refrain from any form of strenuous exercise, caffeine, supplements or alcohol at least 24 hours prior to testing. For the Keirin simulation, each participant consumed water ad lib throughout the day while adhering to their normal diet.

\section{Repeated IPC protocol}

Seven days of repeated IPC was selected from previous clinically based research investigating the same principle. $^{8}$ While in the supine position, a manual inflatable cuff (East Shore Medical Supply, Flushing New York, USA) was positioned on the upper leg where the adductor longus muscle attaches to the inguinal ligament. The cuff was $20 \mathrm{~cm}$ wide with the circumference length adjustable from 42 to $57 \mathrm{~cm}$. Participants received four, $5 \mathrm{~min}$ episodes of IPC $(220 \mathrm{~mm} \mathrm{Hg})$ or 'sham' treatment $(20 \mathrm{~mm} \mathrm{Hg})$ interspersed with 5 min of reperfusion per episode, for each leg. ${ }^{5}$ Alternating unilateral occlusion was achieved by alternating the inflatable cuff from left to right leg (when one leg received reperfusion, the other was under ischaemia). ${ }^{23}$ Toe pulse and oxygen saturation were monitored by a handheld pulse oximeter (Vacumed, Ventura, California, USA), with pulse expected to be non-existent during ischaemia and oxygen saturation dropping below $90 \%$.

\section{Biochemical analysis}

Urine sample preparation, analysis, volume correction, and quantification of neopterin, biopterin (oxidative stress), total neopterin (neopterin $+7,8$ dihydroneoppterin; immune system activation) and total biopterin (biopterin $+7,8$ dihydrobiopterin $\quad\left(\mathrm{BH}_{2}\right)+$ tetrahydroobiopterin $\left(\mathrm{BH}_{4}\right)$; nitric oxide/catecholamine synthesis) were conducted as previously described. ${ }^{20} 21$ Briefly, samples were thawed on ice and diluted 1:40 in $20 \mathrm{mM}$ ammonium phosphate, $\mathrm{pH}$ 2.5. For total biopterin and neopterin analysis, $100 \mu \mathrm{L}$ of diluted sample was aliquoted into a separate microcentrifuge tube. Twenty microlitres of acidic iodide $\left(5.4 \% \mathrm{I}_{2} / 10.8 \% \mathrm{KI}\right.$ in $1 \mathrm{M}$ $\mathrm{HCl}$ ) were added to oxidatively convert $\mathrm{BH}_{2}$ and $\mathrm{BH}_{4}$ to biopterin and 7,8-dihydroneopterin to neopterin before incubation for $15 \mathrm{~min}$ in the dark at room temperature. Following incubation, $10 \mu \mathrm{L}$ of $0.6 \mathrm{M}$ ascorbic acid was added to reduce the tri-iodine and $100 \mu \mathrm{L}$ was transferred to a high-performance liquid chromatography (HPLC) phial for strong cation exchange chromatography analysis (Luna $5 \mu \mathrm{m}$ SCX $100 \AA$, $250 \times 4.6 \mathrm{~mm}$ column (Phenomenex, California, USA)) on a Shimadzu LC-20A with UV detector and autosampler, RF-20A florescence detector and online degasser, with a $20 \mathrm{mM}$ ammonium phosphate $\mathrm{pH} 2.5$ mobile phase pumped isocratically at $1 \mathrm{~mL} / \mathrm{min}$. Biopterin and neopterin were detected at $353 \mathrm{~nm}$ excitation and $438 \mathrm{~nm}$ emission. The concentration and identity of biopterin and neopterin were compared with their corresponding standard (freshly prepared each day and spaced evenly throughout each analytical run) and quantified by peak area using the software Shimadzu Class VP.

\section{Statistical analysis}

The effects of repeated IPC on the change in aerobic and anaerobic capacity and performance, anaerobic capacity parameters during the simulated Keirin competition, and urinary analytes were tested in a linear mixed-effects model fitted with restricted maximum likelihood, conducted in the lme4 package in R V.3.1.1. Between-group and within-group changes were calculated with $\mathrm{p}$ values using Satterthwaite's method of denominator synthesis, conducted in the lmerTest package for $\mathrm{R}$ and supported with Cohen's effect sizes (d) to evaluate the degree of change. Effect sizes were interpreted using thresholds of $0.2,0.6,1.2$ and $>2.0$ for small, moderate, large and very large, respectively. ${ }^{24}$ Changes are reported in comparison with baseline for each dependent variable, with each analysed as the response variable in a separate model. All data are presented as mean \pm SEM, except for the anaerobic capacity and performance parameter figures where the between-participant variability has been removed according to Cousineau. ${ }^{25}$ Significance was set at $\mathrm{p}<0.05$.

\section{RESULTS}

All 18 participants completed their respective treatment and undertook the follow-up testing; hence, all results have been analysed.

\section{Anaerobic measures}

Comparisons between pre-IPC and the best $30 \mathrm{~s}$ Wingate of the Keirin simulation (figure 1D-F) reveal peak power increased $11 \%(\mathrm{p}<0.001, \mathrm{~d}=0.34)$, average power $4.3 \%(\mathrm{p}=0.02, \mathrm{~d}=0.16)$ and fatigue index $12.1 \%$ $(p=0.01, d=0.79)$ in the IPC group, while no changes were observed for the sham group for any of these parameters. Both groups reported a greater perceived feeling of fatigue $30 \mathrm{~min}$ following the first Keirin Wingate test despite no significant changes in lactate measures for either group between the pre-IPC and first Keirin Wingate test (see online supplementary table 3).

\section{Aerobic measures}

Maximal aerobic capacity increased $9.5 \% 48$ hours post IPC treatment with a further increase to $12.8 \%$ above baseline after an additional 7 days $(\mathrm{p}<0.01, \mathrm{~d}=0.91$, 
A

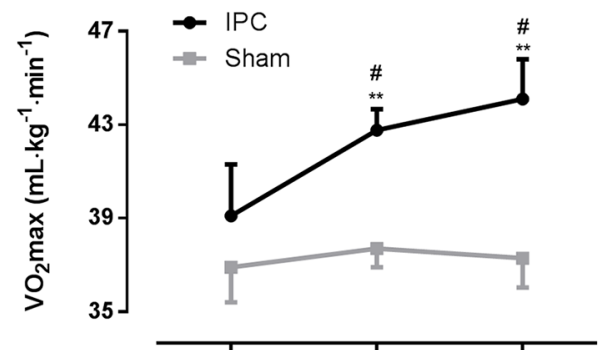

B

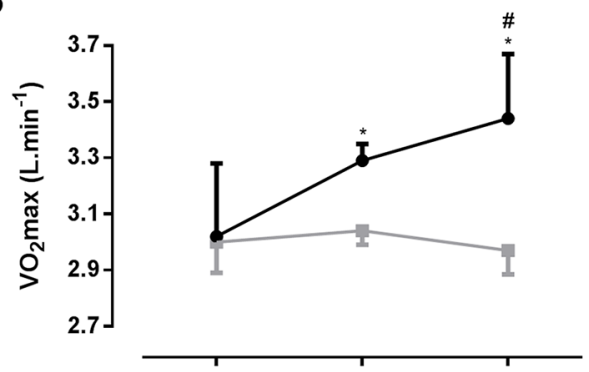

C

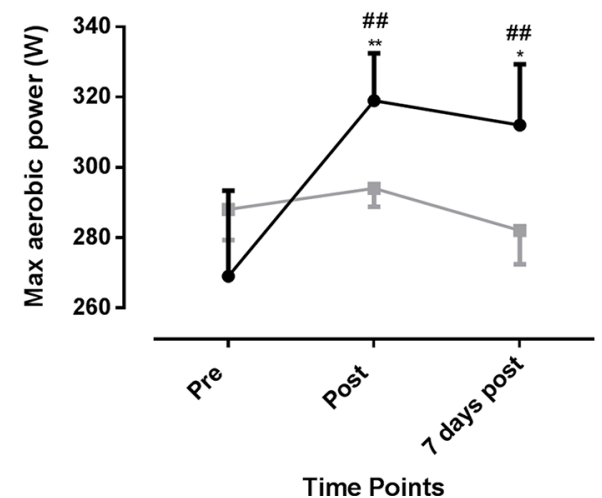

D

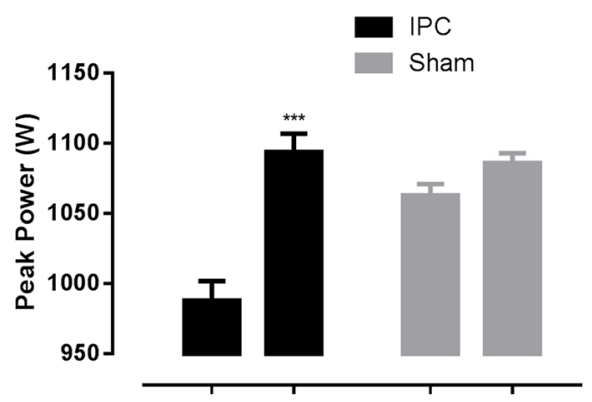

$\mathbf{E}$

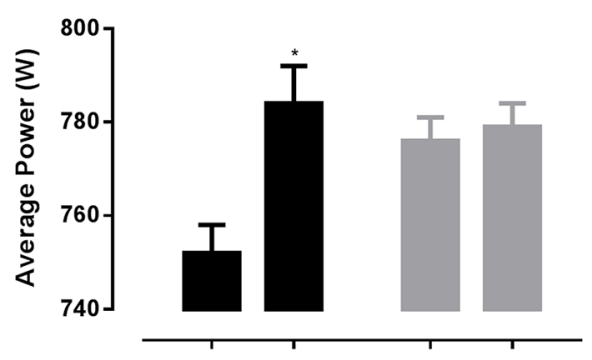

$\mathbf{F}$

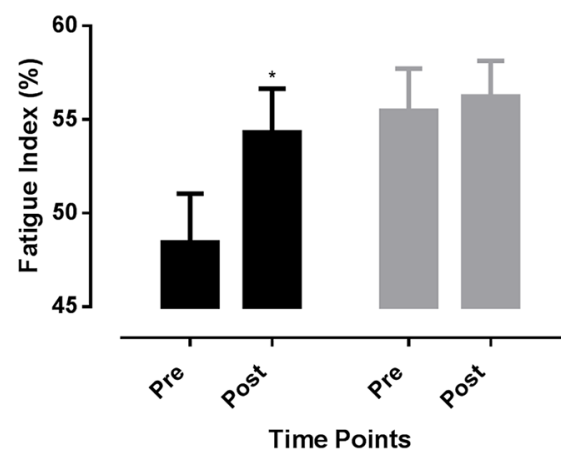

Figure 1 Changes in relative VO2max (A), absolute VO2max (B), maximum aerobic power (C), peak power (D), average power (E) and fatigue index (F) for the repeated ischaemic preconditioning (IPC) and 'sham' groups (sham $=40 \mathrm{~min} /$ day at $20 \mathrm{~mm} \mathrm{Hg}$, $\mathrm{IPC}=40 \mathrm{~min} /$ day at $220 \mathrm{~mm} \mathrm{Hg}$ ). Data are mean+SEM. ${ }^{*}, \# \mathrm{p}<0.05,{ }^{* *}, \# \# \mathrm{p}<0.01,{ }^{* \star *} \mathrm{p}<0.001$; ${ }^{*}$ within group and \#between groups.

moderate effect), as illustrated in figure 1A, B. The IPC treatment also showed a small $(\mathrm{p}<0.01, \mathrm{~d}=0.46)$ effect on increasing maximal aerobic power, which peaked $18.5 \%$ above baseline 48 hours postintervention before regressing back slightly to $16.1 \% 7$ days later (figure 1C). No significant differences were found for maximal heart rate or respiratory exchange ratio (RER) values in either group during any of the VO2max tests (see online supplementary table 1).

\section{Biochemical measures during IPC}

Repeated IPC treatment resulted in significant changes and differences between groups in oxidative stress markers $(\mathrm{p}<0.05)$, with large effect sizes $(\mathrm{d}=1.91$; $\mathrm{d}=1.82$ ) for the weekly increase in biopterin and neopterin, respectively (figure 2). Both groups displayed large $(d=1.91$ and $d=1.31)$ within-group reductions $(47 \%$ and $36 \%)$ in total biopterin for the IPC $(\mathrm{p}<0.01)$ and sham $(\mathrm{p}<0.05)$ groups, respectively.

\section{Keirin event simulation}

Compared with the first Keirin Wingate test, the IPC group significantly increased their peak power by $8.7 \%$ in tests 3 and $4(\mathrm{p}<0.001, \mathrm{~d}=0.14)$, while there were no significant changes in the 'sham' group (figure 3A). Average power had a significant but trivial increase for Wingate 3 and $4(\mathrm{p}<0.05, \mathrm{~d}<0.10)$ when compared with Wingate 1 and 2 (3.2\% and 3.4\%, respectively) for the IPC group, but no changes were observed for the 'sham' group (figure 3B). While the IPC group's fatigue index was elevated during tests 3 and 4 $(\mathrm{p}<0.01, \mathrm{~d}=0.34)$, only Wingate test 3 resulted in significantly greater $(p<0.05, d=0.33)$ levels of fatigue for the 'sham' protocol (figure 3C). 

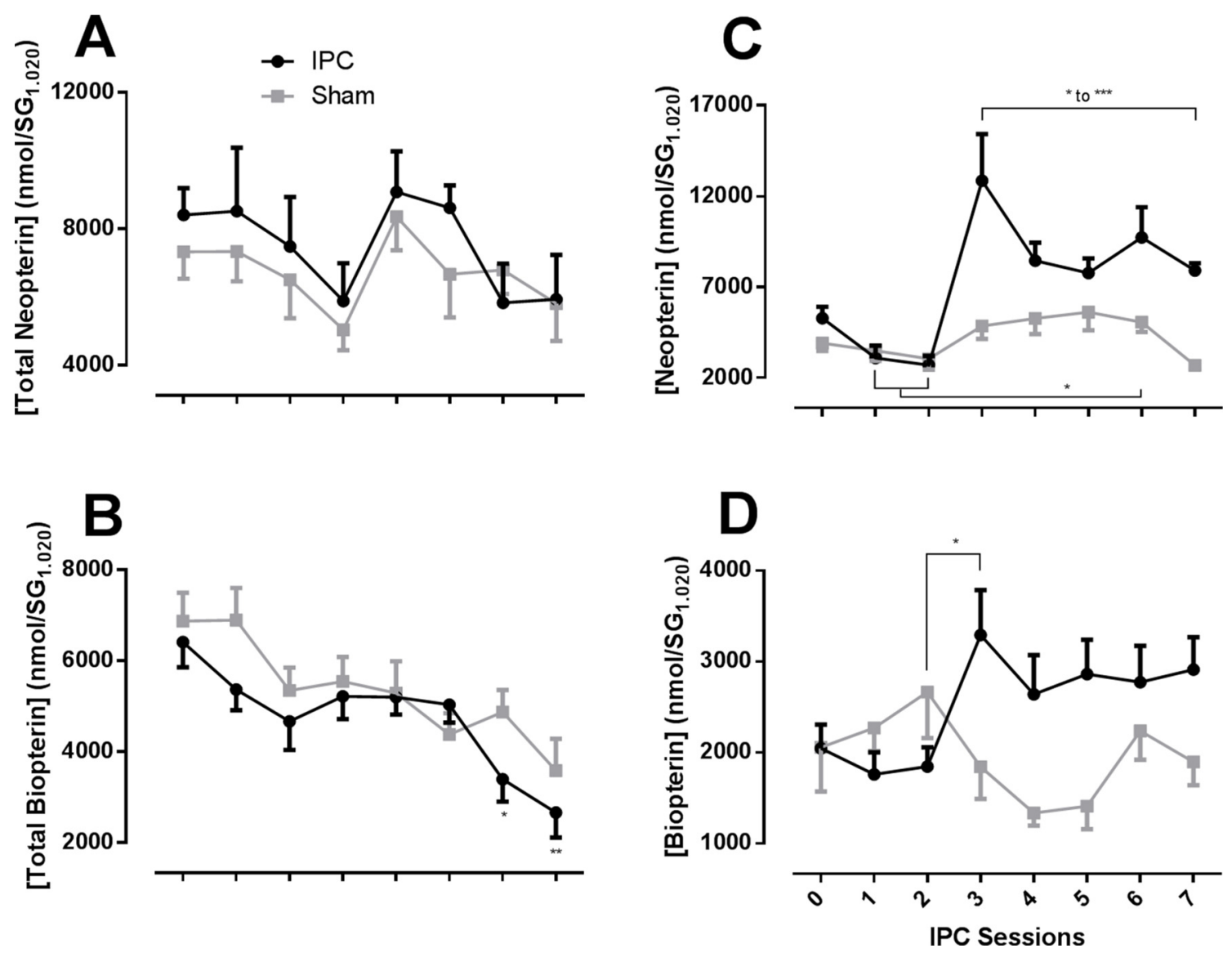

Figure 2 Concentration of urinary biochemical markers during 7 days of ischaemic preconditioning (IPC) for the IPC and 'sham' groups, including total neopterin (A), total biopterin (B), neopterin (C) and biopterin (D). ${ }^{\star} p<0.05,{ }^{\star \star} p<0.01,{ }^{\star \star \star} p<0.001$.

Ratings of perceived fatigue showed similar withingroup trends as both groups experienced increased levels of fatigue $(\mathrm{p}<0.01)$ at specific time points. The 'sham' group had higher blood lactate concentrations prior to test $2(\mathrm{p}<0.05, \mathrm{~d}=0.67)$, while no other statistical differences were observed for either group.

Total neopterin transiently increased following Wingate 2 for the IPC group $(\mathrm{p}=0.02-0.04, \mathrm{~d}=0.39$ 0.42 ), while sham treatment resulted in sustained elevations above baseline at several time points $(\mathrm{p}<0.05$, $\mathrm{d}=0.33-0.37$ ) (figure 3D). This resulted in significant differences between groups before Wingate 3 commenced $(\mathrm{p}=0.04, \mathrm{~d}=0.35)$. IPC resulted in moderate total biopterin increases throughout the Keirin ( $\mathrm{p}<0.05$ to $<0.001, \mathrm{~d}=0.66-0.77$ ) (figure $3 \mathrm{E}$ ), with no evident change in the 'sham' group $(\mathrm{p}>0.05)$. These changes resulted in small to moderate absolute differences between groups $(\mathrm{p}<0.05, \mathrm{~d}=0.46-0.60)$ throughout the simulation.

The oxidised compounds neopterin and biopterin showed markedly different responses. Neopterin significantly decreased $(\mathrm{p}<0.01$ to $<0.001, \mathrm{~d}=0.51-$ $0.71)$ during the second half of the Keirin simulation for the IPC group compared with baseline (figure $3 \mathrm{~F})$, while an immediate and sustained increase was observed for the 'sham' group $(\mathrm{p}<0.05$ to $<0.001$, $\mathrm{d}=0.53-0.61$ ), which returned to baseline concentrations during Wingate 3 and 4 (figure $3 \mathrm{~F}$ ) before increasing significantly 24 hours later $(\mathrm{p}<0.01$, $\mathrm{d}=1.1$ ). Consequently, IPC resulted in significantly lower changes $(p<0.05$ to $<0.01, \quad d=0.54-0.66)$ compared with 'sham' throughout the Keirin simulation and 24 hours post $(\mathrm{p}<0.001, \mathrm{~d}=1.51)$. Biopterin significantly increased compared with baseline immediately prior to the second Wingate test (figure $3 \mathrm{G}$ ). Concentrations remained elevated throughout with greater changes observed for the 'sham' group $(\mathrm{p}<0.01-0.001, \mathrm{~d}=0.73-0.81)$ compared with IPC $(\mathrm{p}<0.05$ to $\mathrm{p}<0.001, \mathrm{~d}=0.61-0.83)$. This resulted in statistically greater differences between groups throughout the third Wingate $(\mathrm{p}<0.05, \mathrm{~d}=0.34-0.40)$ and 24 hours post $(\mathrm{p}<0.05, \mathrm{~d}=1.28)$.

\section{CONCLUSIONS}

Utilising repeated daily ischaemia and reperfusion to elicit an improvement in exercise performance has only recently been investigated ${ }^{8}{ }^{26}$ without considering its use as a 'conditioning tool'. The results suggest that seven consecutive days of IPC may provide ergogenic benefit; however, the optimal conditioning required to elicit a performance or physiological adaptation is yet to be investigated and 


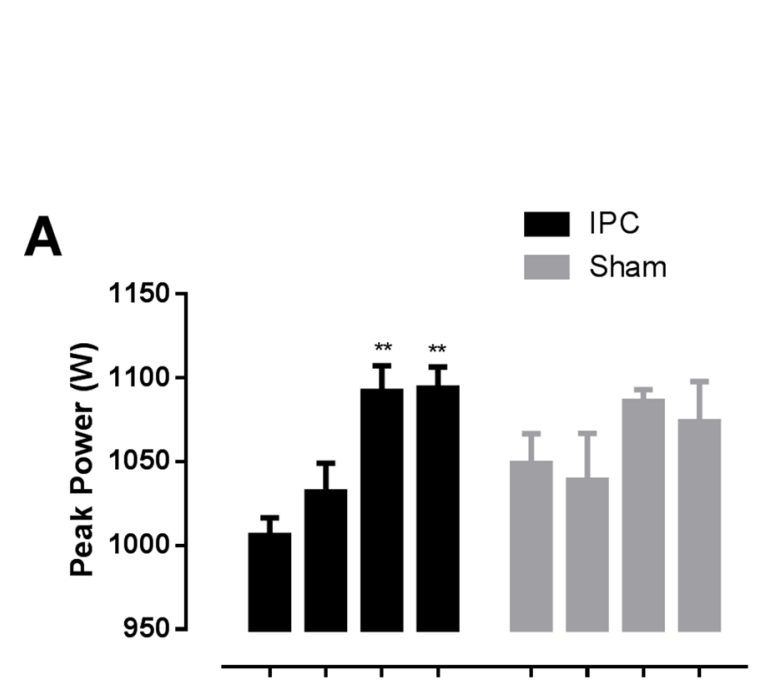

B

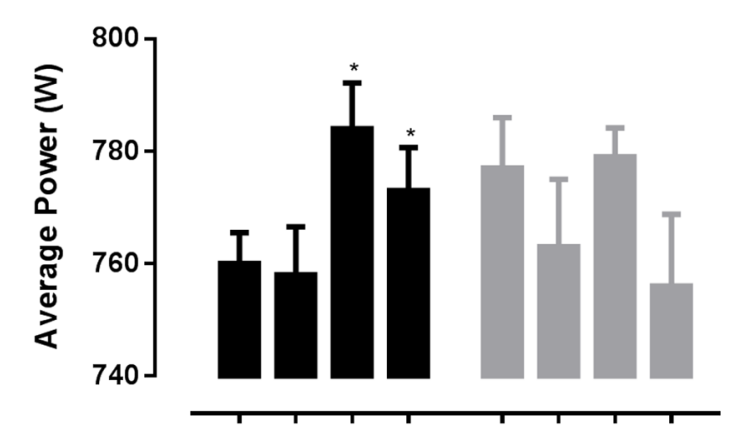

C

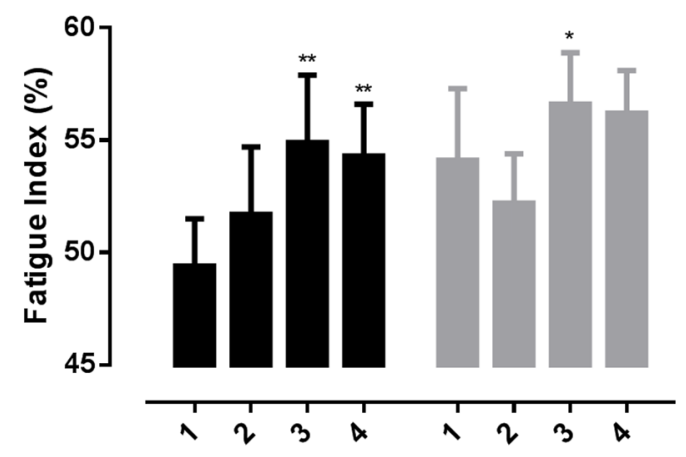

Wingate Number
D

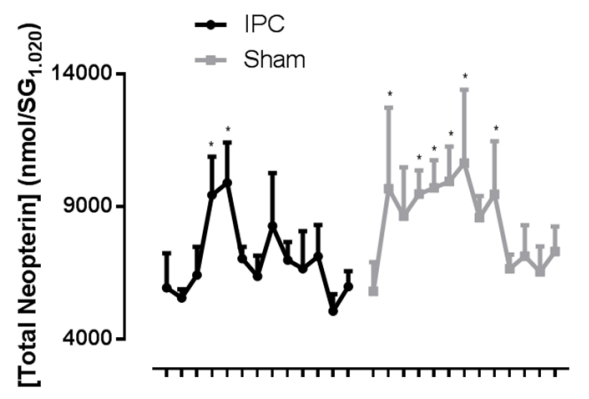

E

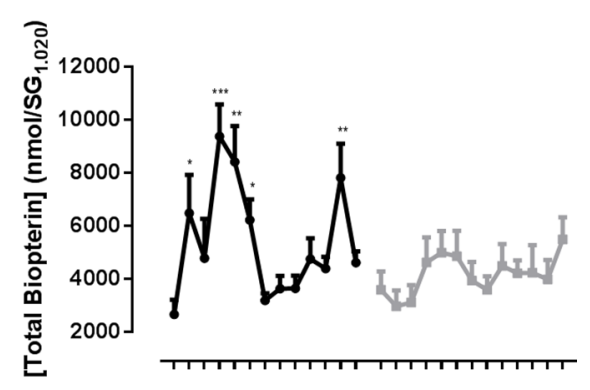

$\mathbf{F}$

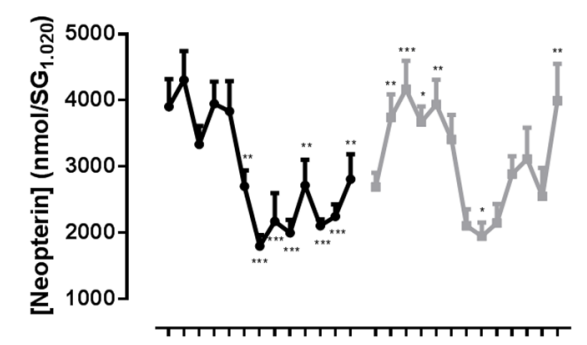

$\mathbf{G}$

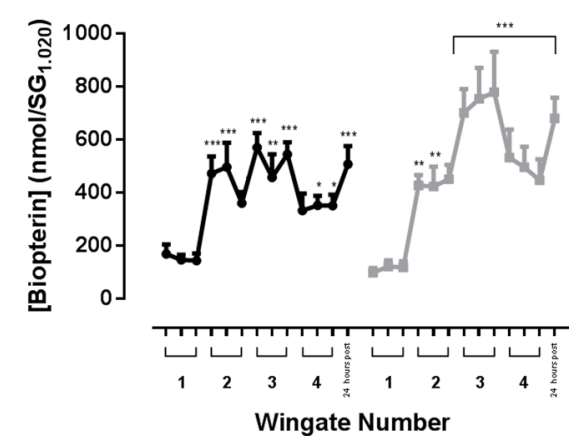

Figure 3 Peak power $(A)$, average power $(B)$, fatigue index $(C)$, total neopterin $(D)$, total biopterin $(E)$, neopterin $(F)$ and biopterin (G) for the repeated ischaemic preconditioning (IPC) and 'sham' protocols during the simulated Keirin competition. Biochemical concentrations were compared with pre-exercise levels. Data are mean $\pm S E M$. ${ }^{\star} p<0.05,{ }^{* \star} p<0.01,{ }^{\star \star \star} p<0.001$.

remains a topic of interest in cardioprotection. ${ }^{6}$ The magnitude of physiological changes seen is substantially greater than reported by de Groot $e t a l^{4}$ and Lalonde and Curnier ${ }^{27}$ who noted that a single application of leg ischaemia improves maximal oxygen consumption by $3 \%$, aerobic power output by $1.6 \%$ and had no change in anaerobic capacity. Our within-treatment group improvement of $12.8 \%$ in maximal oxygen consumption, $18.5 \%$ enhancement in aerobic power, and $11 \%$ and $4.3 \%$ increase in 
peak and average anaerobic power, respectively, looks to justify 7 days of IPC. Yet our data need to be carefully considered in relation to the recreation population recruited, their training status and the psychological factor where participants may have knowingly 'held-back' with the thought of four upcoming bouts. Significant elevations in peak and average power for the IPC group during the Keirin simulation, two critical determining factors in repeated bout anaerobic sport, identify the potential of repeated IPC exposure. These changes are in line with previous IPC research. ${ }^{28}$

We undertook performance testing, 24 and 48 hours post the last IPC session, as practically this fits with when athletes are likely to perform their last exercise sessions during the taper phase leading into a competition. Further study could be undertaken to ascertain if the last IPC session was conducted 72 hours prior to testing and if the repeated IPC effect would still be present.

\section{Biochemical markers}

A review of IPC literature identified a general trend towards aerobic and anaerobic performance improvement but did not elaborate on the physiological or biochemical response during each of the conditions. $^{29}$ It is well-established that IPC results in cellular and genetic adaptations providing protection against hypoxic stress ${ }^{30}$ In an exercise-related context where significant elevations in oxidative stress accompany various forms of activity, the adaptation potentially developed throughout repeated IPC may have increased the cells' ability to modulate the physiological stress response and more efficiently improve exercise capacity and performance through oxidative stress-dependent mechanisms. ${ }^{15}$ The finding that only neopterin and biopterin biomarkers were increased over the week while undertaking the IPC treatment indicates that while oxidative stress was increased, there was minimal immune system activation or nitric oxide/catecholamine synthesis. We speculate either enhanced radical scavenging, a change in the type of oxidant generated or increased oxidative stress. Considering both pterins increased and Zhou et $a l^{31}$ noting oxidative stress is a necessity for IPC-induced protection, a change in the oxidant: antioxidant ratio seems like a plausible scenario. The lack of change in biomarkers of immune system activation is in further accordance with $\mathrm{Tu}$ et $a l,{ }^{15}$ where repetitive IPC attenuated increases in inflammatory mediators.

Repeated IPC resulted in lower levels of indirect markers of oxidative stress during the Keirin simulation. This may explain the ability to maintain a high peak power and reduce the decrement in average power over successive sprints. Increased Reactive Oxygen Species (ROS) such as those produced during high-intensity exercise ${ }^{32}$ has been shown to modulate skeletal muscle force production, ${ }^{33}$ while immune system activation is known to detrimentally affect the same mechanical properties. ${ }^{34}$ It could be postulated that the smaller perturbations in inflammation and oxidative stress experienced in the IPC group had a smaller impact on the contractile properties of the skeletal muscles, allowing for more sustainable and repeatable anaerobic performance.

Repeated IPC also resulted in a significant elevation in urinary total biopterin, suggesting increased vasodilation and sympathetic activation as previously noted. ${ }^{14}$ This may increase oxygen delivery and saturation within the exercising skeletal muscle tissue for enhancement of contractile function, which was also observed between successive bouts. ${ }^{35}$ Horiuchi et al ${ }^{13}$ confirmed this potential theory through improved tissue oxygenation during sympathetic activation at rest and during moderate exercise following IPC. The likely increase in vasodilation and subsequent oxygen delivery in conjunction with previously reported increased blood vessel density in response to repeated IPC-initiated hypoxic conditions ${ }^{36}$ may provide an explanation for the improvement in aerobic capacity.

With the possibility that acute or singular sessions of IPC may improve physiological variables and sporting performance, coaches will likely prescribe IPC multiple times over a set period, thereby using IPC as a conditioning tool. Studies have already begun to investigate the use of IPC over extended periods from 7 days ${ }^{8}$ to 8 weeks. ${ }^{10}$ Future studies should consider investigating multiple IPC sessions per day and use of IPC over time periods longer than 7 days, with different dosages of repeated IPC. The optimal days before competition to implement repeated IPC to maximise any benefit needs to be elucidated.

Repeated IPC over seven consecutive days increased aerobic and anaerobic capacity and anaerobic exercise performance in recreational individuals. The changes were associated with modulations in specific biomarkers of oxidative stress, inflammation and nitric oxide/neurotransmitter synthesis.

\section{Summary}

- substantially improved aerobic and anaerobic capacity

- improvement in certain anaerobic capacity parameters over four successive anaerobic bouts

- significant elevations in neopterin and biopterin during repeated ischaemic preconditioning application that were attenuated in conjunction with total neopterin during repeated anaerobic exercise

- substantially elevated concentrations in total biopterin during repeated anaerobic exercise

Collectively, these findings suggest that ischaemia/ reperfusion associated with alternating unilateral repeated IPC provides a physiological stimulus 
capable of improving both aerobic capacity and anaerobic performance while simultaneously affecting several physiological mechanisms in recreationally active participants.

Acknowledgements The authors would like to thank Larissa Paes for her significant contribution to the preparation of this study, Dr Geoff Shaw for his contribution towards the concept design and each of the participants for their involvement.

Contributors AL manuscript preparation, revision, statistics, interpretation, study design, testing. CP manuscript preparation, interpretation, revision, study design, testing. GB study design, testing, manuscript proofing. HF subject recruitment, testing, manuscript proofing. GP biochemical analysis, manuscript proofing. NS biochemical analysis, manuscript proofing. SPG study design, results interpretation, manuscript proofing.

Funding This research was funded by the School of Health Sciences and School of Biological Sciences, University of Canterbury, Christchurch, New Zealand.

\section{Competing interests None declared.}

Ethics approval University of Canterbury, Human Ethics Committee.

Provenance and peer review Not commissioned; externally peer reviewed.

Open Access This is an Open Access article distributed in accordance with the Creative Commons Attribution Non Commercial (CC BY-NC 4.0) license, which permits others to distribute, remix, adapt, build upon this work noncommercially, and license their derivative works on different terms, provided the original work is properly cited and the use is non-commercial. See: http:// creativecommons.org/licenses/by-nc/4.0/

(c) Article author(s) (or their employer(s) unless otherwise stated in the text of the article) 2017. All rights reserved. No commercial use is permitted unless otherwise expressly granted.

\section{REFERENCES}

1 Faulkner SH, Ferguson RA, Gerrett N, et al. Reducing muscle temperature drop after warm-up improves sprint cycling performance. Med Sci Sports Exerc 2013;45:359-65.

2 Gouvéa AL, Fernandes IA, César EP, et al. The effects of rest intervals on jumping performance: a meta-analysis on post-activation potentiation studies. J Sports $\mathrm{Sci}$ 2013;31:459-67.

3 Barbosa TC, Machado AC, Braz ID, et al. Remote ischemic preconditioning delays fatigue development during handgrip exercise. Scand J Med Sci Sports 2015;25:356-64.

4 de Groot PC, Thijssen DH, Sanchez M, et al. Ischemic preconditioning improves maximal performance in humans. Eur $J$ Appl Physiol 2010;108:141-6.

5 Patterson SD, Bezodis NE, Glaister M, et al. The effect of ischemic preconditioning on repeated sprint cycling performance. Med Sci Sports Exerc 2015;47:1652-8.

6 Sharma V, Marsh R, Cunniffe B, et al. From protecting the heart to improving athletic performance - the benefits of local and remote ischaemic preconditioning. Cardiovasc Drugs Ther 2015;29:573-88.

7 Incognito AV, Burr JF, Millar PJ. The effects of ischemic preconditioning on human exercise performance. Sports Med 2016;46:531-44.

8 Jones H, Hopkins N, Bailey TG, et al. Seven-day remote ischemic preconditioning improves local and systemic endothelial function and microcirculation in healthy humans. Am J Hypertens 2014;27:918-25.

9 Luca MC, Liuni A, McLaughlin K, et al. Daily ischemic preconditioning provides sustained protection from ischemia-reperfusion induced endothelial dysfunction: a human study. J Am Heart Assoc 2013;2:e000075.

10 Jones H, Nyakayiru J, Bailey TG, et al. Impact of eight weeks of repeated ischaemic preconditioning on brachial artery and cutaneous microcirculatory function in healthy males. Eur J Prev Cardiol 2015;22:1083-7.

11 Whittaker P, Przyklenk K. From Ischemic Conditioning to 'Hyperconditioning': Clinical Phenomenon and Basic Science Opportunity. Dose-Response 2014;12:dose-response.1-63.
12 Kleikers PW, Wingler K, Hermans JJ, et al. NADPH oxidases as a source of oxidative stress and molecular target in ischemia/ reperfusion injury. $J$ Mol Med 2012;90:1391-406.

13 Horiuchi M, Endo J, Thijssen DH. Impact of ischemic preconditioning on functional sympatholysis during handgrip exercise in humans. Physiol Rep 2015;3:e12304.

14 Kimura M, Ueda K, Goto C, et al. Repetition of ischemic preconditioning augments endothelium-dependent vasodilation in humans: role of endothelium-derived nitric oxide and endothelial progenitor cells. Arterioscler Thromb Vasc Biol 2007;27:1403-10.

15 Tu XK, Yang WZ, Chen JP, et al. Repetitive ischemic preconditioning attenuates inflammatory reaction and brain damage after focal cerebral ischemia in rats: involvement of PI3K/Akt and ERK1/2 signaling pathway. J Mol Neurosci 2015;55:912-22.

16 de Lucas RD, Caputo F, Mendes de Souza K, et al. Increased platele oxidative metabolism, blood oxidative stress and neopterin levels after ultra-endurance exercise. J Sports Sci 2014;32:22-30.

17 Daubner SC, Le T, Wang S. Tyrosine hydroxylase and regulation of dopamine synthesis. Arch Biochem Biophys 2011;508:1-12.

18 Firth CA, Laing AD, Baird SK, et al. Inflammatory sites as a source of plasma neopterin: measurement of high levels of neopterin and markers of oxidative stress in pus drained from human abscesses. Clin Biochem 2008;41:1078-83.

19 Fuchs D, Weiss G, Wachter H. Neopterin, biochemistry and clinical use as a marker for cellular immune reactions. Int Arch Allergy Immunol 1993;101:1-6.

20 Lindsay A, Healy J, Mills W, et al. Impact-induced muscle damage and urinary pterins in professional rugby: 7,8-dihydroneopterin oxidation by myoglobin. Scand J Med Sci Sports 2016;26:329-37.

21 Lindsay A, Janmale T, Draper N, et al. Measurement of changes in urinary neopterin and total neopterin in body builders using SCX HPLC. Pteridines 2014;25:53-63.

22 Price DD, McGrath PA, Rafii A, et al. The validation of visual analogue scales as ratio scale measures for chronic and experimental pain. Pain 1983:17:45-56.

23 Kraus AS, Pasha EP, Machin DR, et al. Bilateral Upper Limb Remote Ischemic Preconditioning Improves Anaerobic Power. The Open Sports Medicine Journal 2015;9:1-6.

24 Batterham AM, Hopkins WG. Making meaningful inferences about magnitudes. Int J Sports Physiol Perform 2006;1:50-7.

25 Cousineau D. Confidence intervals in within-subject designs: A simpler solution to Loftus and Masson's method. Tutor Quant Methods Psychol 2005;1:42-5.

26 Banks L, Wells GD, Clarizia NA, et al. Short-term remote ischemic preconditioning is not associated with improved blood pressure and exercise capacity in young adults. Appl Physiol Nutr Metab 2016;41:903-6.

27 Lalonde F, Curnier DY. Can anaerobic performance be improved by remote ischemic preconditioning? J Strength Cond Res 2015;29:80-5.

28 Bailey TG, Jones $\mathrm{H}$, Gregson W, et al. Effect of ischemic preconditioning on lactate accumulation and running performance. Med Sci Sports Exerc 2012;44:2084-9.

29 Salvador AF, De Aguiar RA, Lisbôa FD, et al. Ischemic preconditioning and exercise performance: a systematic review and meta-analysis. Int J Sports Physiol Perform 2016;11:4-14.

30 Semenza GL. Hypoxia-inducible factor 1: regulator of mitochondrial metabolism and mediator of ischemic preconditioning. Biochim Biophys Acta 2011;1813:1263-8.

31 Zhou X, Zhai X, Ashraf M. Direct evidence that initial oxidative stress triggered by preconditioning contributes to second window of protection by endogenous antioxidant enzyme in myocytes. Circulation 1996;93:1177-84.

32 Alessio HM, Hagerman AE, Fulkerson BK, et al. Generation of reactive oxygen species after exhaustive aerobic and isometric exercise. Med Sci Sports Exerc 2000;32:1576-81.

33 Reid MB, Khawli FA, Moody MR. Reactive oxygen in skeletal muscle. III. Contractility of unfatigued muscle. J Appl Physiol 1993;75:1081-7.

34 Powers SK, Jackson MJ. Exercise-induced oxidative stress: cellular mechanisms and impact on muscle force production. Physiol Rev 2008;88:1243-76.

35 French DN, Kraemer WJ, Volek JS, et al. Anticipatory responses of catecholamines on muscle force production. J Appl Physiol 2007;102:94-102.

36 Kawata $\mathrm{H}$, Yoshida $\mathrm{K}$, Kawamoto $\mathrm{A}$, et al. Ischemic preconditioning upregulates vascular endothelial growth factor mRNA expression and neovascularization via nuclear translocation of protein kinase $C$ epsilon in the rat ischemic myocardium. Circ Res 2001;88:696-704. 